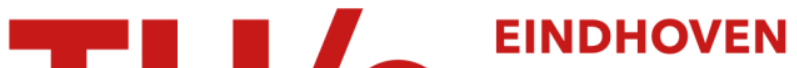 \\ UNIVERSITY OF \\ TECHNOLOGY
}

\section{Glassy boundary layers vs enhanced mobility in capped polymer films}

Citation for published version (APA):

Batistakis, C., Michels, M. A. J., \& Lyulin, A. V. (2013). Glassy boundary layers vs enhanced mobility in capped polymer films. Journal of Chemical Physics, 139(2), 024906-1/9. [024906]. https://doi.org/10.1063/1.4811237

DOI:

10.1063/1.4811237

Document status and date:

Published: 01/01/2013

\section{Document Version:}

Publisher's PDF, also known as Version of Record (includes final page, issue and volume numbers)

\section{Please check the document version of this publication:}

- A submitted manuscript is the version of the article upon submission and before peer-review. There can be important differences between the submitted version and the official published version of record. People interested in the research are advised to contact the author for the final version of the publication, or visit the $\mathrm{DOI}$ to the publisher's website.

- The final author version and the galley proof are versions of the publication after peer review.

- The final published version features the final layout of the paper including the volume, issue and page numbers.

Link to publication

\section{General rights}

Copyright and moral rights for the publications made accessible in the public portal are retained by the authors and/or other copyright owners and it is a condition of accessing publications that users recognise and abide by the legal requirements associated with these rights.

- Users may download and print one copy of any publication from the public portal for the purpose of private study or research.

- You may not further distribute the material or use it for any profit-making activity or commercial gain

- You may freely distribute the URL identifying the publication in the public portal.

If the publication is distributed under the terms of Article 25fa of the Dutch Copyright Act, indicated by the "Taverne" license above, please follow below link for the End User Agreement:

www.tue.nl/taverne

Take down policy

If you believe that this document breaches copyright please contact us at:

openaccess@tue.nl

providing details and we will investigate your claim. 


\title{
Glassy boundary layers vs enhanced mobility in capped polymer films
}

\author{
C. Batistakis, ${ }^{1,2, a)}$ M. A. J. Michels, ${ }^{1}$ and A. V. Lyulin ${ }^{1}$ \\ ${ }^{1}$ Theory of Polymers and Soft Matter (TPS), Department of Applied Physics, Technische Universiteit \\ Eindhoven, P.O. Box 513, 5600 MB Eindhoven, The Netherlands \\ ${ }^{2}$ Dutch Polymer Institute, P.O. Box 902, 5600 AX Eindhoven, The Netherlands
}

(Received 5 March 2013; accepted 31 May 2013; published online 12 July 2013)

\begin{abstract}
Molecular-dynamics simulations have been carried out for a coarse-grained model of a random ABcopolymer confined between two crystalline substrates. The strength of substrate-polymer interactions, and the distance between the two substrates have been varied in a wide range. For thick films the film-averaged segmental mobility decreases for intermediate adsorption strengths, but start to increase for very high substrate-polymer attraction strength. We saw that this non-monotonic behavior is caused by a very strong heterogeneity of the segmental dynamics above the glass-transition temperature: the segmental mobility slows down drastically close to adsorbing substrates, but strongly increases in the middle part of the film. This effect, and its sensitive dependence on film thickness, are explained by finite-size effects in confinement, in combination with glassy boundary layers. It is demonstrated that film-averaged mobility as often measured cannot be understood without resolving local mobility in space and time. (C) 2013 AIP Publishing LLC. [http://dx.doi.org/10.1063/1.4811237]
\end{abstract}

\section{INTRODUCTION}

Thin polymer films, usually supported by one substrate or capped between two surfaces, can be found in various technological applications. ${ }^{1-8}$ Examples include coatings, lithographic devices, organic photovoltaics (OPVs), and polymer nanocomposites such as tires, seals, etc., where filler particles in polymer matrices can be connected through ultrathin polymeric bridges. ${ }^{7,8}$ For film thicknesses less than few nanometers, i.e., comparable to the size of the individual polymer coil, these films exhibit significant changes in their dynamical behavior when compared with the bulk. ${ }^{9-19}$ These changes depend on the adsorption strength to substrates, but they can also be attributed to the finite-size scaling effects when the films are extremely thin and the polymer chains are accordingly strongly confined.

The majority of the research on thin amorphous polymer films has focused on the film-thickness dependence of the glass-transition temperature $T_{g}$. Various experiments on supported polymer films of nanoscopic dimensions have shown deviations in the glass-transition $\left(T_{g}\right)$ temperature compared with the bulk. Keddie et al.,,${ }^{9,10}$ by using ellipsometry, have measured reductions in $T_{g}$ both for polystyrene (PS) films supported on hydrogen-terminated silicon surfaces and PMMA films supported by different substrates. $T_{g}$ reduction was also reported by Forrest et al., ${ }^{11}$ where by using Brillouin light scattering, the authors found a linear decrease of $T_{g}$ with film thickness for ultrathin (less than few nanometers) freestanding PS films of high molecular weights. Soles et al. ${ }^{12}$ showed that in ultrathin polycarbonate (PC) films on silicon-oxide substrates, the specific method used for the $T_{g}$ determination can give contradictory results. When determining $T_{g}$ by structural techniques, like Specular X-ray

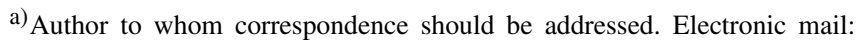
c.batistakis@tue.nl
}

Reflectivity (SXR) and Positron Annihilation Lifetime Spectroscopy (PALS), they found that $T_{g}$ decreases with film thickness, whereas measurements by inelastic neutron scattering (INS), which measures the atomic mean-square displacements, showed a slight increase of $T_{g}$ for extremely thin films. This anomalous behavior was also found for PS films on silicon substrates, ${ }^{13}$ where ellipsometry measurements showed a reduction of $T_{g}$ with film thickness, ${ }^{9,13}$ whereas measurements with INS showed an increase.

Generally, the majority of the studies indicate a reduction of $T_{g}$ upon confinement, i.e., when the film thickness becomes comparable to the radius of gyration of the individual polymer chain. Such a reduction leads finally to an increased segmental mobility at fixed temperature. The segmental mobility can be layer-dependent, being different at different distances from the substrates. The value of $T_{g}$ itself is a rather indirect measure of some average polymer segmental mobility, and, as stated above, proves to be technique-dependent. Calorimetry, rheology, ellipsometry, and spectroscopy methods provide different $T_{g}$ values, which has resulted in much controversy in the field.

In addition to the many experimental studies on the $T_{g}$ deviations from the bulk in ultrathin polymer films, molecular simulations have been performed. They can provide direct access to the dynamical behavior at the nanometer scale. They also offer the advantage of easily measuring and handling the effects of the substrate surfaces, free interfaces, substrate-polymer interactions, etc., which cause strong inhomogeneities in the film dynamics. Torres et al. ${ }^{15}$ measured the film-thickness dependence of $T_{g}$ for films supported by attractive walls. They saw that $T_{g}$ is fairly constant for thick films but can positively or negatively deviate when decreasing film thickness, depending on the wall attraction strength. Layer-resolved analysis of the dynamical behavior of confined 1,4-polybutadiene between attracting graphite walls showed a slowing down of the polymer mobility close 
to the substrates. ${ }^{16}$ The same was found for atactic short-chain polysterene films capped between attracting gold surfaces, where a dramatic slowing down of the dynamics close to the substrates was observed, when compared with the bulk. ${ }^{19}$ Inhomogeneous dynamics for strongly confined supported PS films was also found by Hudzinskyy et al., ${ }^{17}$ who measured the $T_{g}$-values distribution across different film layers. They showed that the film-averaged $T_{g}$ based on density results from a subtle interplay of several effects that may either raise or decrease it from the bulk value. Varnik et al. ${ }^{14}$ simulated films of different thicknesses which were capped between two smooth and repulsive walls. The walls caused strongly inhomogeneous dynamical behavior inside the film, while a decreasing film thickness led to a decrease of the film-averaged mode-coupling critical temperature $T_{c}$ from its bulk value. Varnik et al. worked the concept by Adam and Gibbs ${ }^{20}$ of collectively rearranging regions (CRRs) near the glass transition. In their argument the thickness of a capped film acts as a cut-off length for the growing cluster size $\xi$ of the CRRs when the film cools down, leading to a maximum for the cluster relaxation time $\tau_{c l} \sim \xi^{\nu}$. By finite size scaling, the authors explained their observation that thinner films observed faster averaged dynamics.

In our previous publication ${ }^{18}$ we studied the dynamical behavior of coarse-grained polymer films of different thicknesses but of the same averaged density. These films were capped between attractive crystalline substrates. We used weak and energetically neutral substrate-polymer interactions of a simple Lennard-Jones (LJ) type. Two competing effects were found: in thick films (much larger than the individualchain radius of gyration), the segmental mobility slows down close to attractive substrates when compared with the middle of the films, but the overall mobility accelerates when decreasing film thickness (stronger confinement). The latter was understood by the same finite-size scaling argument as used by Varnik et al. ${ }^{14}$

We conclude that all previous experimental and simulation results have shown that various parameters, such as temperature, local density, the strength of adsorption of the polymer to the substrates, or the extent of confinement can strongly affect the dynamics of ultra-thin polymer films in opposite ways. Nevertheless, the relative importance of these parameters for motion on different length scales is still unclear. In the present study we extend our previous model for a wider range of substrate-polymer interactions and film thicknesses. Our main goal is to investigate whether finite-size scaling effects and the accompanying accelerated dynamics remain important in cases of very strong strengths of adsorption of the polymers to the substrates. For that purpose, we simulated coarse-grained films of various thicknesses, from comparable to the individual-chain radius of gyration and up to several times larger. The substrate attraction strength was varied by two orders of magnitude, while the average film density was held constant at all simulated temperatures (NVT ensemble). Thick films were also created and studied under the application of a constant normal pressure (NPT ensemble).

The paper is organized as follows. In Sec. II we give details about the model as well as a description of the sample preparation and equilibration procedure. In Sec. III A 1 the effect is shown of increasing the substrate-polymer interaction strength on the segmental dynamics of a film with a constant thickness. For that purpose, the thickest simulated film has been chosen, in which a well-defined middle part is not directly affected by the substrate attraction. Layer-resolved analysis of the orientational and translational film dynamics follows in Sec. III A 2. In Sec. III B the segmental film dynamics under constant external pressure is investigated in different film layers, and the results are compared with those of the NVT simulations of Sec. III A 2. In Sec. III C the importance is shown of finite-size scaling effects on the dynamics of thinner films for high substrate-polymer interaction strengths. In Sec. III D, an overall picture is given of dependence on thickness and adsorption strength of the characteristic translational relaxation time. Finally, in Sec. IV a summary and conclusions are presented.

\section{MODEL AND SIMULATION DETAILS}

We simulate coarse-grained thin polymer films that are capped between two attractive crystalline substrates. Polymer chains are represented with the help of a bead-rod model. Each chain consists of 50 monomers, with $80 \%$ of type A and $20 \%$ of type B (differing in their sizes and masses, see below) and all in random positions along the chain. Every film was created from 100 non-entangled polymer chains. Each of two substrates consist of three layers of LJ spheres in an FCC arrangement. All bonded monomer pairs are connected with rigid bonds and all non-bonded monomer pairs interact via a truncated LJ potential:

$$
V_{L J}(r)= \begin{cases}4 \epsilon\left[\left(\frac{\sigma}{r}\right)^{12}-\left(\frac{\sigma}{r}\right)^{6}\right] & \text { if } r \leq r_{c} \\ 0 & \text { if } r>r_{c}\end{cases}
$$

with a cutoff at $r_{c}=2.5 \sigma$.

All quantities are expressed in LJ units, where $\sigma, \epsilon$, $m$, and $\tau=\sigma(m / \epsilon)^{1 / 2}$ are the units of length, energy, mass, and time, respectively. The temperature is expressed in units of $\epsilon / k_{B}$, the density in units of $m / \sigma^{3}$, and the pressure in units of $\epsilon / \sigma^{3}$. For the type-A monomers we chose $m=m_{A}$, $\sigma=\sigma_{A}$, and $\epsilon=\epsilon_{A A}$, and for the type-B monomers $\epsilon_{B B}=\epsilon_{A A}, \sigma_{B}=1.2 \sigma_{A}$, and $m_{B}=(1.2)^{3} m_{A}$. Concerning the substrate particles, the LJ parameters were chosen to be $\sigma_{s}=0.85$ and $\epsilon_{s} \gg 1$. For all the non-bonded interactions, the $\sigma_{i j}$ (i.e., the $\sigma_{A B}$ and $\sigma_{s p}$ ) were calculated according to Lorentz-Berthelot rule $\sigma_{i j}=\frac{1}{2}\left(\sigma_{i i}+\sigma_{j j}\right)$, as well as the $\epsilon_{A B}$, where $\epsilon_{A B}=\sqrt{\epsilon_{A A} \epsilon_{B B}}$, while various values have been chosen for the interaction strength $\epsilon_{s p}$ in the range 0.1-10.

The effects of the confinement and the adsorption to the substrates have been studied for films of various thicknesses $D$ in the range 5-30 and for a wide range of substrate-polymer interaction parameters $\epsilon_{s p}$, as mentioned above. Each film has been created with the same amount of monomers; hence, larger lateral dimensions were used for thinner films. The averaged density was chosen to be equal to $\rho=1.2$ and was held constant during the equilibration and production procedure. This was achieved by running all simulations in the NVT ensemble, where the temperature coupling was implemented with the help of the Nose-Hoover thermostat; the cho- 

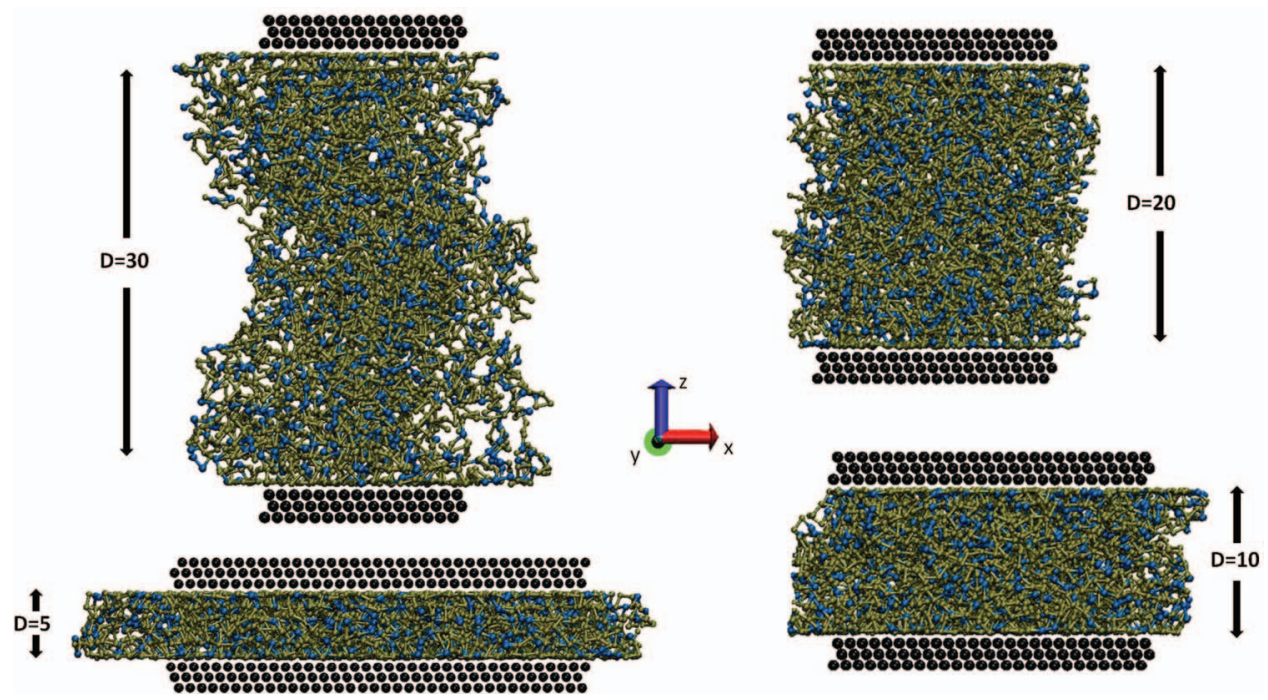

FIG. 1. Snapshots of the equilibrated polymer films of different thicknesses $D$ (in the graph the thicknesses $D=30,20,10$, and 5 are illustrated) capped between two crystalline substrates at high temperature $T=1.5$ and for substrate-polymer interaction strength of $\epsilon_{s p}=1$. All films have the same amount of monomers and the same density $\rho=1.2$. For illustration purposes, the size of the polymer beads has been scaled-down. The different film thicknesses have been achieved by using substrates of different lateral dimensions. Periodic-boundary conditions hold in all three dimensions. The illustration of the systems has been achieved with the help of the molecular visualization program visual molecular dynamics (VMD). ${ }^{24}$

sen time constant was $\tau_{t}=0.5 \tau$. The reason for choosing such a high density is to ensure that the internal pressure in all films is positive at all temperatures. Periodic boundary conditions were applied in all directions.

We performed all simulations with the open-source molecular-dynamics (MD) simulation package GROMACS ${ }^{21}$ (versions 4.5.4 and 4.5.5). The integration of Newton's equations was done with the leapfrog variant of the velocity Verlet algorithm. The time step was chosen to be $\Delta t=0.001 \tau$ at high temperatures, in the view of the large thermal fluctuations, although it was increased to $\Delta t=0.005 \tau$ at lower temperatures $(T \leq 1.5)$. For the constraining of the bonds, the P-lincs algorithm was used. ${ }^{22,23}$

\section{A. Equilibration and cooling procedures}

All films have been created and equilibrated during $10^{4} \tau$ at the rather high temperature $T=5$ (well above the bulk $T_{g}$ $\approx 1$ of this high-density model). In the first stage of the equilibration, the substrate-polymer interaction was chosen to be purely repulsive to avoid immediate substrate-polymer contacts. This was achieved by using only the repulsive part of the Lennard-Jones potential (Eq. (1)) with $\epsilon_{s p}=1$,

$$
V_{L J}=4 \epsilon_{s p}\left(\frac{\sigma}{r}\right)^{12}
$$

At the end of this stage of equilibration the filmaveraged individual-chain radius of gyration was calculated as $R_{g} \approx 3.3$ for all film thicknesses, and the fluctuations around this value were less than 5\%. More details about the filmscreation methods and the validity of the equilibration procedure can be found in our previous publication. ${ }^{18}$ In the next stage of the equilibration procedure, the substrate-polymer interactions were described with the full Lennard-Jones potential (Eq. (1)) for different interaction strengths $\epsilon_{s p}=0.1-10$.
For each value of $\epsilon_{s p}$, the films were again equilibrated at high temperature $T=5$ for sufficiently long time (10000-20000 $\tau$ ). Finally, all films were cooled down to $T=0.5$ with a constant cooling rate of $0.0003 \frac{\epsilon / k_{B}}{\tau}$. An example of the created films is shown in Figure 1.

\section{RESULTS AND DISCUSSION}

\section{A. Effect of the adsorption-strength increase at constant film thickness}

\section{Film-averaged segmental dynamics}

The effect of the substrate-polymer interaction strength $\epsilon_{s p}$ on the film-averaged segmental dynamics has been studied by calculating the mean-square translational displacements (MSTD) for chain segments, averaged over all monomers and all chains,

$$
d(t)^{2}=\left\langle\Delta \bar{r}^{2}\right\rangle,
$$

where $\Delta \bar{r}=\bar{r}(t)-\bar{r}(0)$.

The results for film thickness $D=30$ and for different interactions strengths $\epsilon_{s p}$, together with bulk values at the same temperature $T=1$, are shown in Figure 2.

Qualitatively similar behavior is observed for all substrate adsorption strengths. At short times we distinguish the ballistic regime with slope 2 . As has been predicted by the mode-coupling theory (MCT), ${ }^{25,26}$ the ballistic regime is followed by a plateau, where each tagged particle is temporarily trapped in a cage created by its neighbors (the so-called cage effect). The time needed for a monomer to escape from this cage is the so-called $\alpha$-relaxation time $\left(\tau_{\alpha}\right)$. As soon as the monomer escapes from the cage, the subdiffusive Rouse regime follows with an average slope of 0.57 , a value which is slightly larger than the theoretical Rouse exponent 0.5. This difference can be attributed to the short length of the simulated polymer chains. Indeed, simulations with shorter 


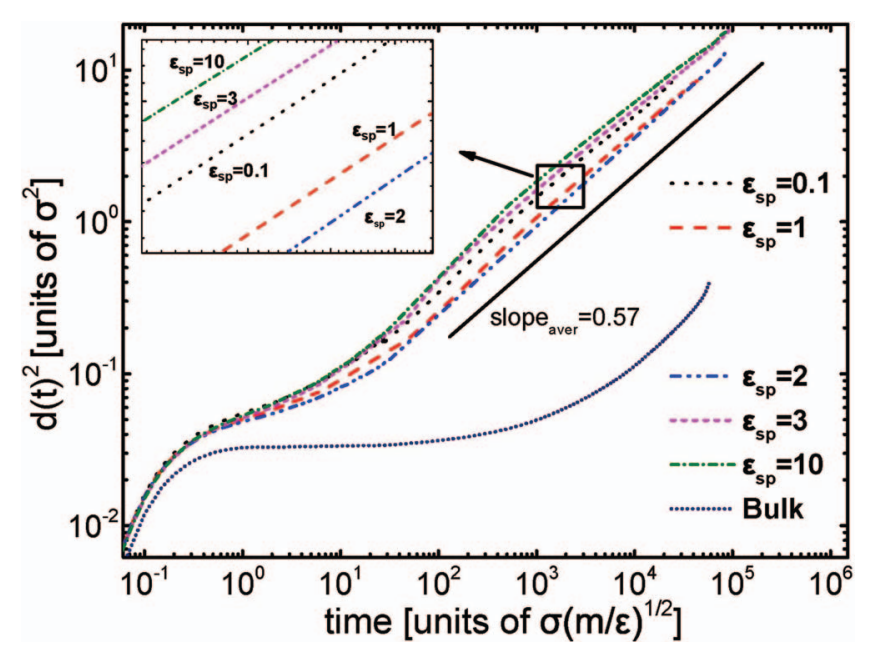

FIG. 2. Sample-averaged mean-squared segmental translational displacements at temperature $T=1$, for the bulk polymer (short-dotted line) and for the film with thickness $D=30$ and different substrate-polymer interaction strengths $\epsilon_{s p}$ (dotted, dashed, dashed-dotted-dotted, short-dashed, and short-dashed-dotted lines correspond to attraction strengths $\epsilon_{s p}=0.1,1,2$, 3 , and 10 , respectively). The inset shows the non-monotonic dynamics with increasing $\epsilon_{s p}$.

chains have shown even larger differences between the average slope of the subdiffusive Rouse regime and the theoretical Rouse exponent. ${ }^{27}$ In Figure 2, it is seen that for all simulated strengths of adsorption, the translational mobility of the confined films is accelerated compared to the bulk. This acceleration can be explained from finite-size scaling effects in confined films. ${ }^{14,18}$ The comparison of the translational mobility shows that increasing the substrate attraction from $\epsilon_{s p}=0.1$ to $\epsilon_{s p}=2$ leads to an increase of the filmaveraged $\alpha$-relaxation time, and a slowing down of the filmaveraged segmental dynamics. However, the effect is not monotonic; further increase of the adsorption strength (i.e., to $\left.\epsilon_{s p}=3-10\right)$ leads to subsequent acceleration of the filmaveraged dynamics.

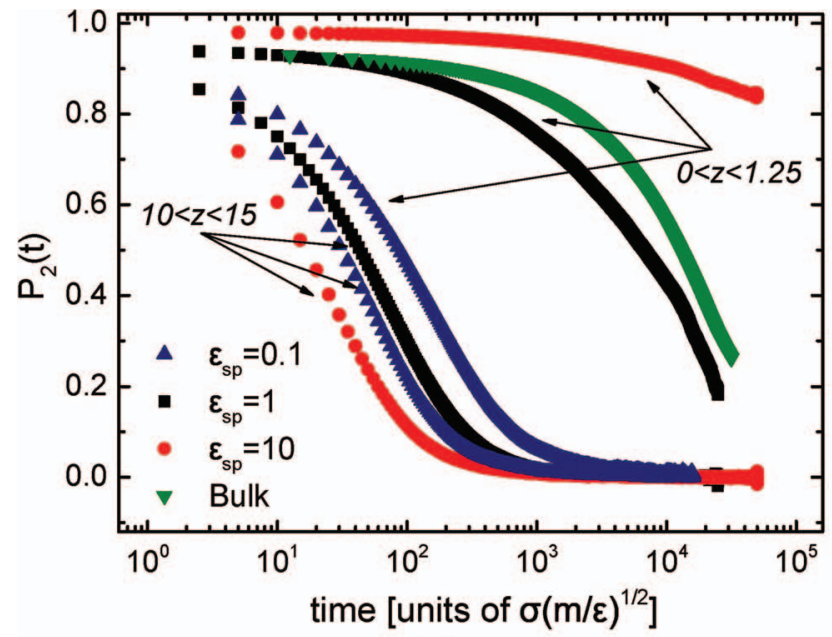

(a)

\section{Layer-resolved segmental dynamics}

In order to have a better insight in the observed nonmonotonic change of segmental mobility, the segmental dynamics for film thickness $D=30$ has been analysed in different film layers and for all considered substrate-polymer interaction strengths $\epsilon_{s p}$. This analysis was done by studying both the MSTD and the segmental orientational mobility; for the later we used the Legendre second-order autocorrelation function $(\mathrm{ACF})$

$$
P_{2}[\vec{b}(0) \cdot \vec{b}(t)]=\left\langle\frac{3}{2}(\vec{b}(0) \cdot \vec{b}(t))^{2}-\frac{1}{2}\right\rangle,
$$

where $\vec{b}$ is the bond vector between two neighboring beads as measured at time 0 and time $t$. The brackets denote a time averaging as well as an averaging over all the bonds which belong to a specific layer of fixed width $l=1.25 \sigma$. We defined that a bond belongs to a specific layer if the middle of the bond is inside that layer at time $t=0$. With the chosen layer width, the full relaxation of the majority of the bonds takes place inside the layer. ${ }^{18}$ In Figure 3(a) we see an example of the $P_{2}$ ACF behavior in a semilog plot for the layers closest to the substrates $(0<z<1.25$, where $z$ denotes the distance from the nearest substrate) and for those in the middle of the film $(10<z<15)$ for a wide range of substratepolymer interaction strengths $\left(\epsilon_{s p}=0.1-10\right) . P_{2}(t)$ decays faster in the middle of the film than close to the substrates for all adsorption strengths. Increasing the substrate-polymer interaction strength from $\epsilon_{s p}=0.1$ to $\epsilon_{s p}=1$ leads to a slower decay of the ACF in all film regions, although further increase from $\epsilon_{s p}=1$ to $\epsilon_{s p}=10$ resulted to even slower decay close to the substrates, but, surprisingly, faster decay is observed in the middle of the film. A similar picture was obtained when calculating the layer-resolved segmental mean-square translational displacements (Figure 3(b)), thus providing a qualitative matching of the translational and orientational mobility of the system.

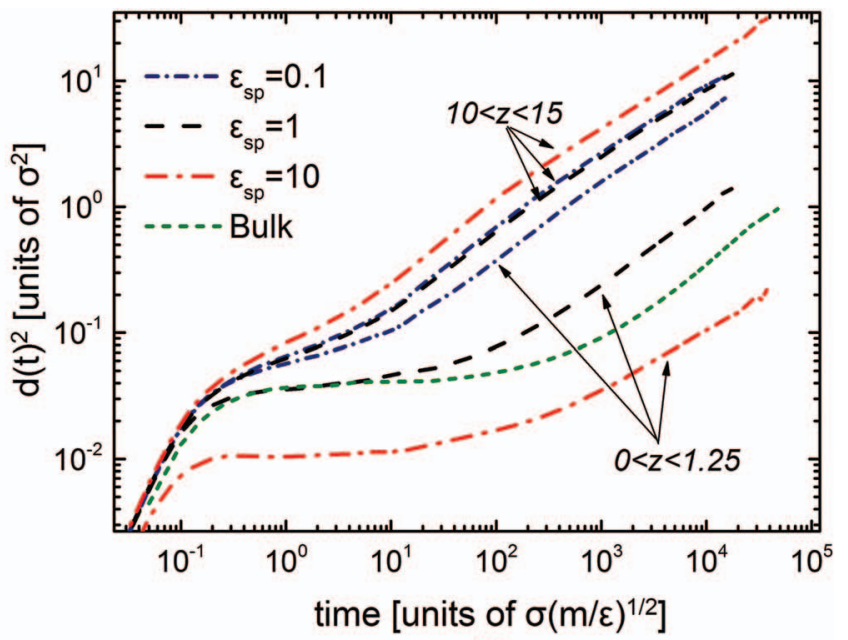

(b)

FIG. 3. (a) Decay of the $P_{2}(t) \mathrm{ACFs}$ for the layers closest to the substrates $(0<z<1.25)$ and for the middle layers $(10<z<15)$ of the film with thickness $D$ $=30$ and at temperature $T=1.1 ; z$ is the distance from the nearest substrate. The blue triangles, black squares, red dots, and green inverse triangles correspond to substrate attractions $\epsilon_{s p}=0.1,1,10$, and to the bulk, respectively. (b) Mean-square translational displacements for the same regions and temperature as in (a). The short-dashed-dotted, dashed, dashed-dotted, and short-dashed lines correspond to substrate attractions $\epsilon_{s p}=0.1,1,10$, and to the bulk, respectively. 


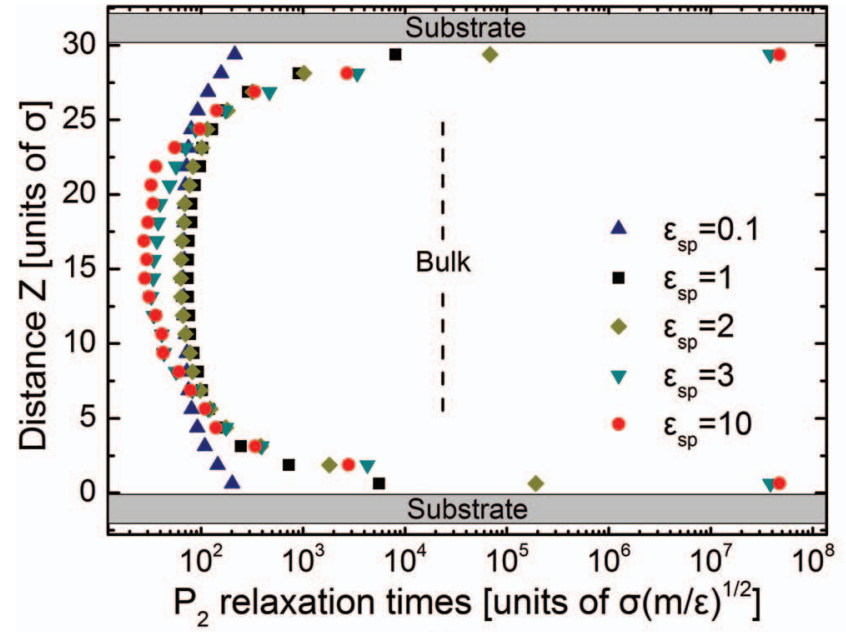

(a)

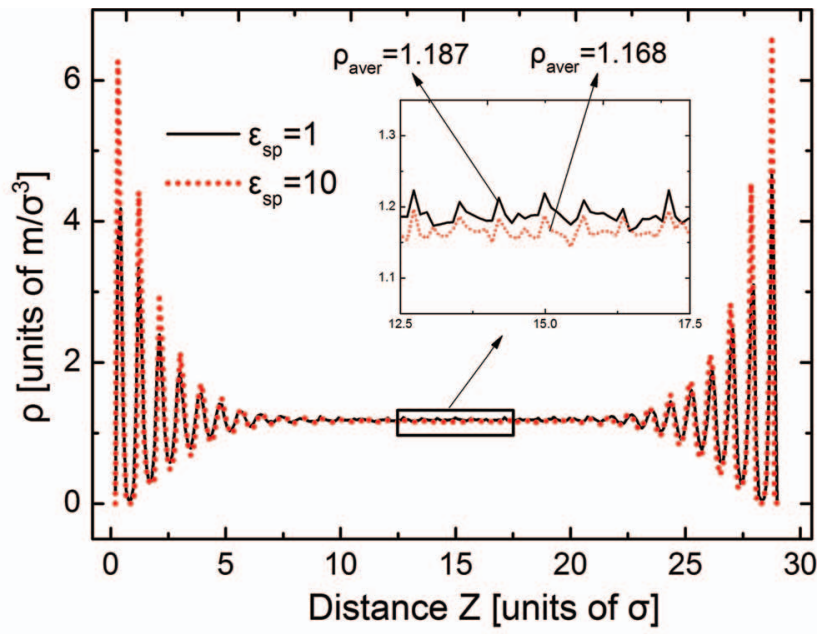

(b)

FIG. 4. (a) Characteristic relaxation times resulting from layer-resolved orientational segmental mobility for film thickness $D=30$ at temperature $T=1.1$ and for substrate-polymer interaction strengths $\epsilon_{s p}=0.1-10$. The vertical dashed line shows the bulk value at the same density $\rho=1.2$ as in the films and at the same temperature. (b) Density profiles for film thickness $D=30$ and for substrate attraction strengths $\epsilon_{s p}=1$ (black line) and $\epsilon_{s p}=10$ (red-dotted line) at $T$ $=1.1$. The inset depicts the density differences in the middle region $(12.5<z<17.5)$. The average middle-densities were calculated to be equal to $\rho=1.187$ when $\epsilon_{s p}=1$ and $\rho=1.168$ when $\epsilon_{s p}=10$.

The characteristic relaxation times of the orientational relaxation have been extracted by fitting the final parts of the $P_{2}(t)$ ACFs with the Kohlrausch-Williams-Watts (KWW) stretched-exponential function ${ }^{28}$

$$
P_{2}(t)=\alpha \exp \left(-\left(\frac{t}{\tau}\right)^{\beta}\right),
$$

where $\alpha \leq 1, \tau$ is the characteristic relaxation time, and $\beta$ shows the non-exponential nature of the relaxation process. The results are summarized in Figure 4(a). A slowing down of the segmental mobility is observed close to the substratepolymer interface $(0<z<5)$, when compared with the middle of the films $(10<z<15)$. This effect is directly connected to the substrate attraction strength, with a stronger slowing down near the substrates for higher values of $\epsilon_{s p}$. The characteristic relaxation times in the middle of the films $(10<z$ $<15$ ) are fairly constant for $\epsilon_{s p}=0.1,1$, or 2 , but a significant shift to lower values was found for $\epsilon_{s p}=3$ and 10. This nonmonotonic behavior is in qualitative agreement with the results of Figure 2 for the film-averaged translational segmental mobility. Indeed, when increasing the strength of adsorption from $\epsilon_{s p}=0.1$ to $\epsilon_{s p}=10$, a strong decrease of the segmental mobility in the layers close to the substrates is initially insufficiently compensated, but the acceleration observed in the middle layers (the larger fraction of the film), beyond the adsorption strength $\epsilon_{s p}=2$ is able to give an overall increase of the film-averaged segmental dynamics, as shown in Figure 2.

Strong inhomogeneities and a layer dependence can also be found in the structural properties of the films. Figure 4(b) shows the density profiles for film thickness $D=30$ and substrate-polymer interactions $\epsilon_{s p}=1$ and $\epsilon_{s p}=10$. Analysis of the profiles shows two well-defined density regimes in the films. In the layers close to the substrates strong ordering is observed and sharp density variations are visible, whereas the middle of the film is characterized by a smooth density profile. The observed monomer ordering close to the substrates is an entropic effect, but strongly depends on the substrate-polymer interaction strength $\epsilon_{s p}$. Increasing $\epsilon_{s p}$ causes sharper density variations but also an increase of the average density in this region. As the total film density was held constant $(\rho=1.2)$ for all the simulated values of $\epsilon_{s p}$, an increase of the density close to the substrates should lead to a decrease of the density in the middle of the film. Indeed, the average densities in the middle layers were calculated for temperature $T=1.1$ and were found equal to $\rho=1.187$ and $\rho=1.168$ for $\epsilon_{s p}=1$ and $\epsilon_{s p}=10$, respectively. These density differences are rather small (see the inset in Figure 4) but not negligible, and might be responsible for the observed acceleration in the dynamics in the middle of the films as found in Figure 4(a). In Sec. III B we argue about the effect of these density differences on the segmental dynamics.

\section{B. NPT-ensemble simulations}

In order to assess the importance of the density differences found in the middle of the thick films with different adsorption strengths (Figure 4(b)) to the segmental dynamics, we have repeated the analysis of Figure 4 for the case of a constant normal external pressure to the films (NPT-ensemble simulations) and for substrate-polymer interaction strengths $\epsilon_{s p}=1$ and $\epsilon_{s p}=10$. The equilibration and production procedures were similar to those described in Sec. II A. During equilibration and cooling-down of the samples, the Berendsen thermostat and barostat were used (NPT ensemble), although the production runs after cooling took place in an NVT ensemble again, using the Nose-Hoover thermostat. The time constants were chosen to be $\tau_{t}=0.5 \tau$ for both thermostats and $\tau_{p}=2.5 \tau$ for the barostat. Application of a constant external pressure leads to decrease of film thickness with decreasing temperature. Therefore, the pressure was adjusted to be $p=15$, so that the film thickness is $D \approx 30$ at temperature $T \approx 1$. 


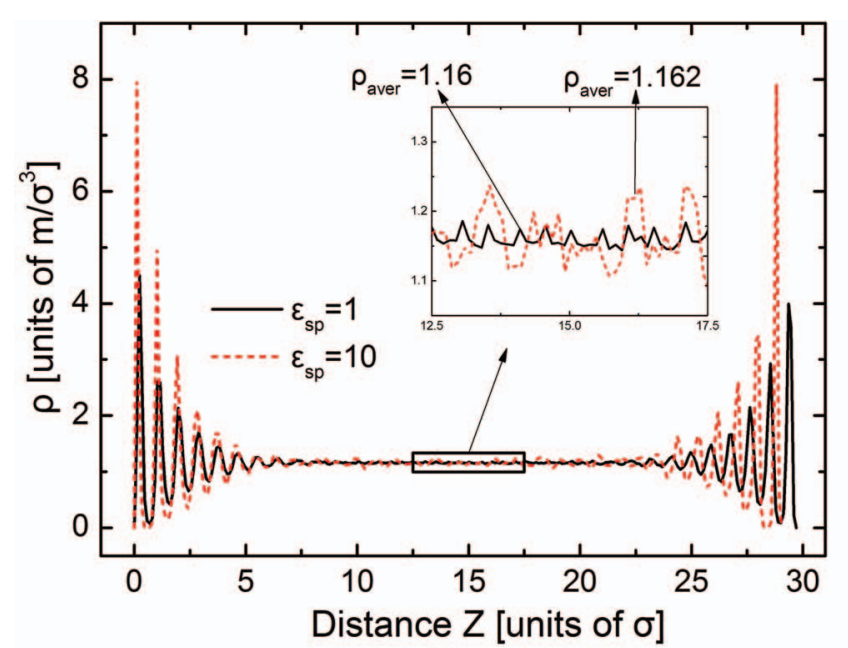

(a)

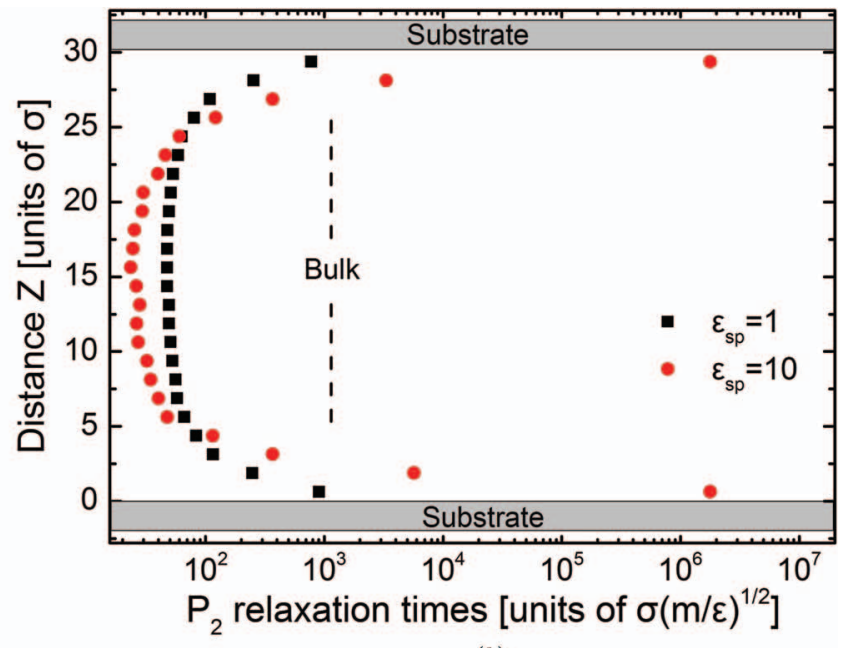

(b)

FIG. 5. (a) Density profiles at temperature $T=1$ for films created under a constant normal pressure $p=15$. The black line corresponds to substrate attraction strength $\epsilon_{s p}=1$, while the red dotted line to $\epsilon_{s p}=10$. The resulting thickness for both cases is $D \simeq 30$. The inset shows the densities in the middle-layers $(12.5<z<17.5)$ in each case. (b) Layer-resolved $P_{2}$ relaxation times for the same films and at the same temperature $T=1$. The black squares correspond to energetically neutral $\left(\epsilon_{s p}=1\right)$ and the red dots to strongly attractive $\left(\epsilon_{s p}=10\right)$ substrates. The vertical dashed line shows the bulk relaxation time at temperature $T=1$, as produced after a constant-volume simulation (NVT-ensemble) at the same density $\rho=1.16$ as in the middle of films.

Figure 5(a) shows the density profiles of these simulated films for temperature $T=1$. An ordering is observed close to the substrates, which is followed by a plateau in the middle of each film; similar behavior was found in the films which were prepared under the same constant density (Figure 4(b)). Increasing the substrate attraction leads to an increase of density close to the substrates and, due to the application of a constant normal external pressure, to an overall increase of the average film density. This can be seen by checking the density profiles of Figure 5(a) in the middle region, where for $\epsilon_{s p}=10$ density differences in the middle of the films were not observed (Figure 5(a), inset), in contrast to the NVT-ensemble simulation results (Figure 4(b), inset).

The $P_{2}$ ACFs have been calculated for different layers of the films and for temperature $T=1$, and the characteristic relaxation times have been extracted following the same procedure as described in Sec. III A 2. The results are summarized in Figure 5(b). Despite the absence of density differences in the middle of the two films, a noticeable shift of the relaxation times to lower values is still observed in this region when increasing the strength of adsorption to the substrates. This is in agreement with the results of Figure 4(a), thus showing the minor importance of the small density differences (Figure 4(b)) to the segmental dynamics. But the origin of the acceleration observed in the middle layers of thick films when increasing the adsorption strength still remains to be explained.

Note that for both simulated adsorption strengths, the middle-layer relaxation times are well below the corresponding bulk values at the same density $\rho=1.16$ as in the middle of the films. This deviation may arise from the fact that the temperature $T=1$ is close enough to the bulk glass-transition temperature, so the bulk relaxation time is strongly influenced. The other reason could be that even the $D=30$ film is not thick enough, and for this high-density model confinement effects are important for all the simulated film thicknesses.
Possibly at higher temperatures the difference between the middle-layer dynamics and the bulk at the same density will be smaller.

\section{Effect of film thickness on segmental dynamics}

As mentioned in the Introduction, previous studies on films confined under repulsive ${ }^{14}$ or energetically neutral ${ }^{18}$ substrates have shown an acceleration of the dynamics when increasing confinement, due to finite-size scaling effects. ${ }^{14,20}$ An example can be seen in Figure 6(a), which depicts the characteristic orientational relaxation times in different film layers for different film thicknesses and for $\epsilon_{s p}=1$. We can now compare this with the local mobility for strongly adsorbing substrates. Figure 6(b) shows the layer-resolved orientational relaxation times for different film thicknesses for the case $\epsilon_{s p}=10$. The comparison of Figures 6(a) and 6(b) reveals different behavior at each film thickness when strongly increasing the substrate attraction. As shown in Sec. III A 2, for thick films $(D=30)$, an about one order of magnitude acceleration of the dynamics in the middle-layers was observed upon stronger adsorption to the substrates and, as was shown in Sec. III B, this effect is not connected to density differences. Similar behavior can be seen for intermediate thicknesses (i.e., $D=20$ ) but the effect is less pronounced. For thin films $(D \leq 10)$, tremendous slowing down of the mobility close to the substrates was found with increasing $\epsilon_{s p}$, while the acceleration in the middle layers is negligible. Therefore, the clear shift to higher overall mobility with decreasing film thickness, as shown for films confined under energetically neutral $\left(\varepsilon_{s p}=1\right)$ substrates, is not found for stronger adsorption strengths. Moreover, the effect of increased substrate attraction strength $\epsilon_{s p}$ on the layer resolved dynamics is found to be thickness-dependent.

The existence of layers with extremely slow dynamics close to strongly adsorbing substrates (especially in the cases 


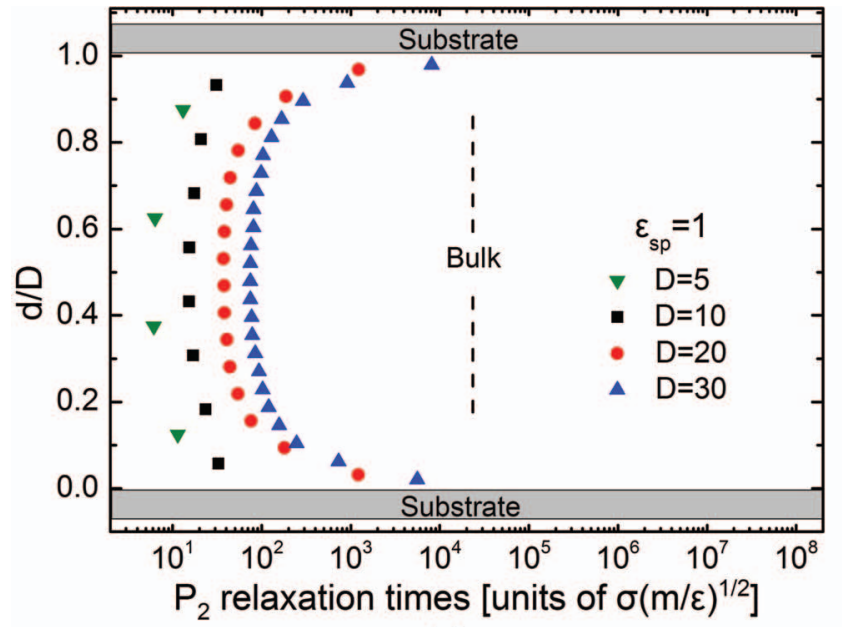

(a)

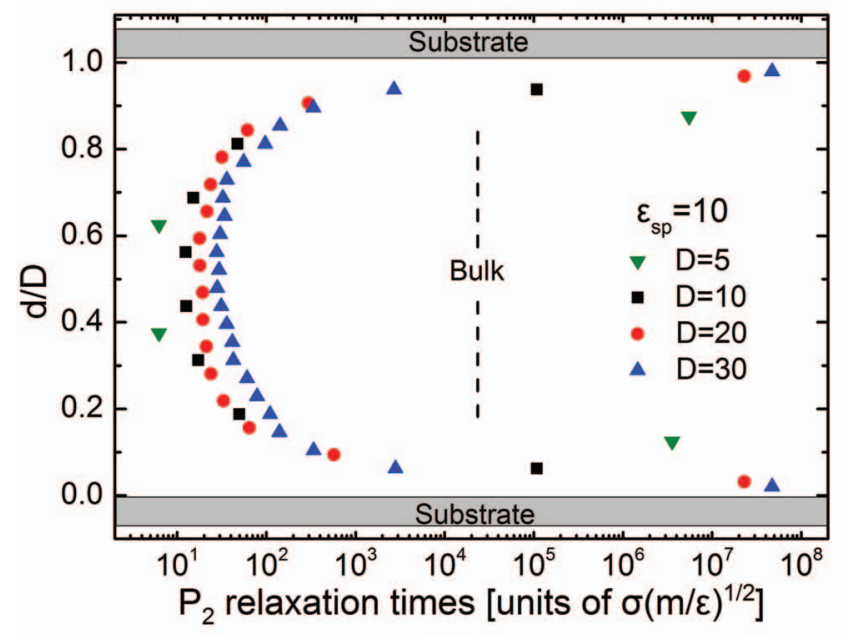

(b)

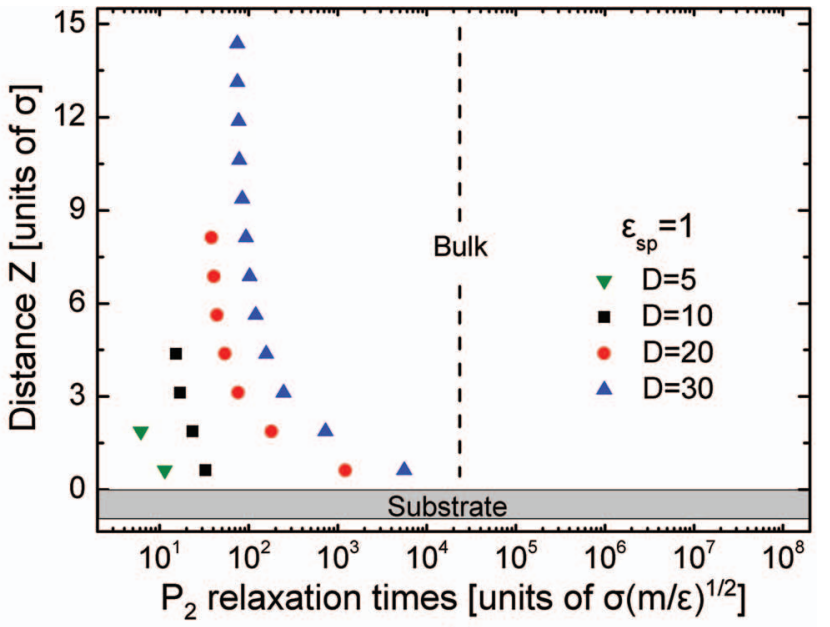

(c)

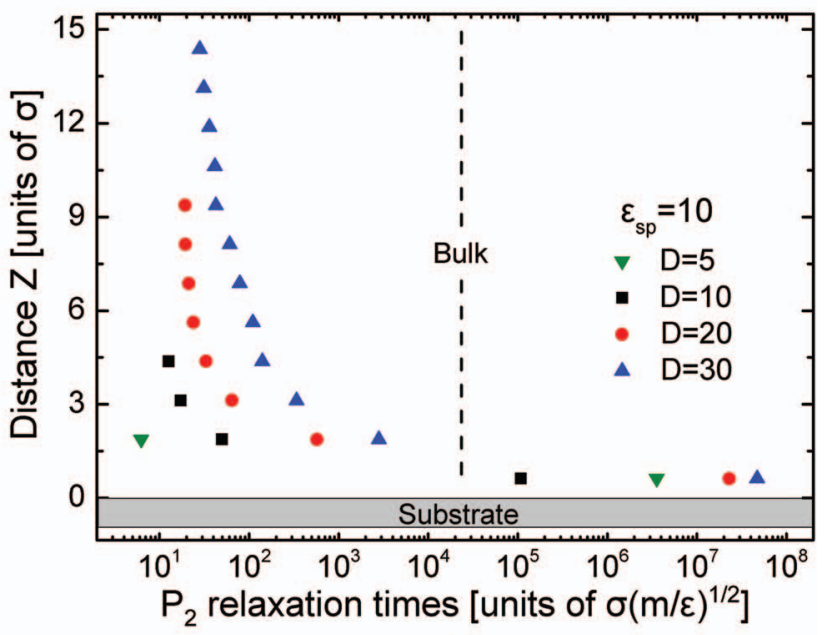

(d)

FIG. 6. (a) Layer-resolved orientational relaxation times for different film thicknesses at temperature $T=1.1$ and for energetically neutral $\left(\epsilon_{s p}=1\right)$ substratepolymer interaction strength. The blue triangles, red dots, black squares, and green inverse triangles correspond to film thicknesses $D=30,20,10$, and 5 , respectively. Vertical dashed line shows the bulk value at the same density and temperature with the films. (b) Similar to (a) but for strongly attractive substrates $\left(\epsilon_{s p}=10\right)$. Note that while the vertical scale have been normalized with $D$, each dot represents an average over a layer thickness of $1.25 \sigma$, independent of film thickness $D$. (c) and (d) are similar to (a) and (b), respectively, but the data are plotted in the range of $0<z<D / 2$ for each film thickness and without normalizing the vertical scales.

of intermediate and high film thicknesses) indicate that part of the films is in the highly mobile melt state and part is in a glassy state. Therefore, we may assume the picture of a high-mobility middle part of the film confined between two glassy substrates, with a glass-melt interaction strength that is energetically neutral $\left(\epsilon_{g m}=\epsilon_{p p}=1\right)$. This may explain the observed accelerated dynamics in the middle layers of thick films with strong substrate adsorption. These melt like middle layers will have a smaller thickness for larger adsorption strength due to thicker glassy layers. Assuming the same finite-size scaling argument as before, we can understand the accelerated dynamics of the middle layers from the effective decrease of their thickness when the strength of adsorption $\epsilon_{s p}$ increases. For very thin films $(D \leq 10)$, i.e., of the order of the polymer gyration radius, the consideration of separate contrasting layers become less meaningful. Stronger confinement then results in faster dynamics, and the slowing-down of the dynamics close to the substrates is far less pronounced. Indeed, the measured $\tau_{\alpha}$ in the layers closest to the substrates $(0<z<1.25$, with $z$ denoting the distance from the nearest substrate) is $1-3$ orders of magnitude smaller than the one measured for thicker films. Therefore we can assume little influence of an increased $\epsilon_{s p}$ on formation of glassy layers in this case, and so explain the negligible effect on the dynamics in the middle of very thin films.

\section{Film-averaged $\alpha$-relaxation vs film thickness}

The above discussed competing effects of the confinement and substrate attraction on the segmental dynamics of capped polymer films resulted in a layer-resolved dynamical behavior that strongly depends on film-thickness and substrate-polymer interaction strength. The impact of these combined effects on the film-averaged dynamics still has to be evaluated.

Figure 7(a) shows the film-averaged mean-square translational displacements (Eq. (3)) for $\epsilon_{s p}=10$ and film thicknesses $D=5-30$ as well as for the bulk. Similar curves were 


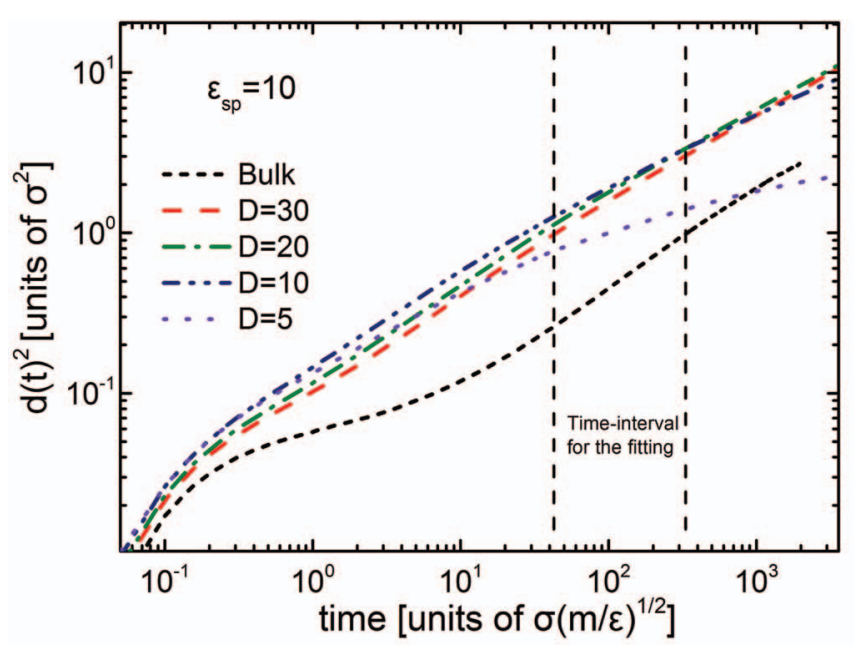

(a)

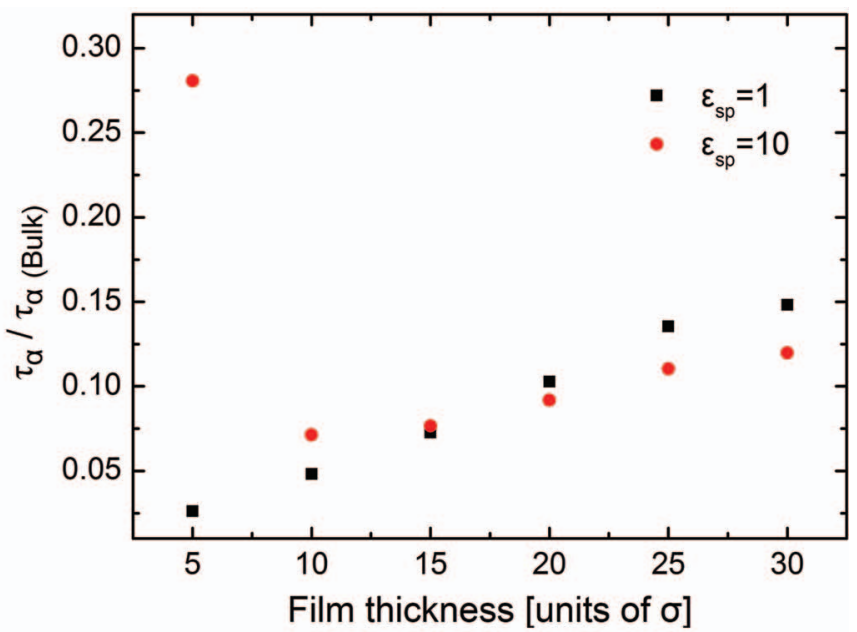

(b)

FIG. 7. (a) Film-averaged mean-square translational displacements at $T=1.5$ for films of thicknesses $D=30,20,10,5$ confined between strongly adsorbing substrates $\left(\epsilon_{s p}=10\right)$ as well as for the bulk. The dashed vertical lines show the chosen time interval for the extraction of the characteristic relaxation time $\tau_{\alpha}$. (b) Evolution of the corresponding characteristic relaxation times $\tau_{\alpha}$ with film thickness for energetically neutral $\left(\epsilon_{s p}=1-\right.$ black squares $)$ and strongly adsorbing substrates $\left(\epsilon_{s p}=10-\right.$ red dots). The times $\tau_{\alpha}$ are normalized with the bulk $\tau_{\alpha}$ at the same density and temperature.

produced for all simulated film thicknesses and for $\epsilon_{s p}=1$. As explained in Sec. III A 1, for each individual particle "cageescape" is followed by the subdiffusive Rouse regime with a constant slope around 0.5 . This behavior can be clearly seen in Figure 7(a) also for a bulk polymer, as well as for thick films ( $D=30$ and $D=20)$, but it is not seen for the average over thinner films, where the slope is changing with time. This change is due to the strong inhomogeneities in the mobility, as shown in Figure 6. Indeed, since the mean-square displacement constitutes an average over very high- and low-mobility layers, the average diffusion is strongly affected. A constant subdiffusive Rouse slope should be found, only when all particles in the film have relaxed. Therefore, the extraction of a characteristic film-averaged relaxation time should depend on the chosen time interval.

To illustrate this, $\alpha$-relaxation times were extracted by fitting parts of all curves for all film thicknesses, over the same time interval, with the power law ${ }^{29,30}$

$$
\left\langle\Delta r^{2}(t)\right\rangle \sim\left(\frac{t}{\tau_{\alpha}}\right)^{\alpha}
$$

We chose the interval between the bulk "cage-escape time" ${ }^{31}$ until the time where the average mean-square displacement for the bulk is $\sigma^{2}$. Figure 7(b) shows the evolution of these relaxation times $\tau_{\alpha}$ with film thickness and for substratepolymer interaction strengths $\epsilon_{s p}=1$ (black-squares) and $\epsilon_{s p}=10$ (red-dots). For films confined between energetically neutral substrates, a monotonic behavior is observed, where decreasing film thickness leads to acceleration of the film-averaged segmental dynamics. For strongly attractive substrates, the dynamical behavior when decreasing film thickness is non-monotonic. The film-averaged translational relaxation time is then decreasing when the thickness decreases from $D=30$ to $D=15$; negligible differences occur for thicknesses $D=15$ and $D=10$, and a sharp increase is found for ultra-thin films $(D=5)$. An explanation of this non-monotonic behavior can be given, based on the results of
Figure 6. For all film thicknesses, increasing the strength of adsorption from $\epsilon_{s p}=1$ to $\epsilon_{s p}=10$ leads to slowing down of the dynamics close to the substrates; but above a certain thickness glassy layers are created and acceleration of the dynamics takes place in the middle of the films. For thicknesses above $D=15$, the averaging of these two competing effects leads to an overall acceleration of the film-averaged dynamics, since the broad middle part dominates. For thicknesses $D=10$ and below, the acceleration in the middle of the films is minor (Figure 6) and therefore, the film-averaged characteristic relaxation time is increasing with increasing $\epsilon_{s p}$. The same effect takes place for ultra-thin films $(D=5)$, where for high $\epsilon_{s p}$ the low-mobility layers constitute the dominant volume fraction and cause a very strong increase of filmaveraged characteristic relaxation time.

\section{SUMMARY AND CONCLUSIONS}

We have performed molecular-dynamics simulations of bead-rod random copolymer chains capped between two attractive crystalline substrates. Different substrate-substrate separations (i.e., film thicknesses) as well as various strengths of substrate-polymer interaction were used. The systems were simulated in a NVT ensemble, therefore, in all cases, the filmaveraged density was constant and equal to $\rho=1.2$. Increasing the strength of adsorption to the substrates in thickest films $(D=30)$ leads to a non-monotonic behavior of the filmaveraged segmental translational mobility (Figure 2). Slowing down was observed when increasing the adsorption strength from $\epsilon_{s p}=0.1$ to $\epsilon_{s p}=2$, but, surprisingly, an acceleration followed for higher values of $\epsilon_{s p}$. For all simulated values of $\epsilon_{s p}$, strong acceleration was found as compared to the bulk, due to finite-size scaling effects. ${ }^{14,18,20}$

The segmental orientational mobility was analyzed for film thickness $D=30$ in different film layers with the help of the $P_{2}(t)$ ACFs (Eq. (4)) and the characteristic relaxation times were extracted. The dynamics in different film layers 
were found to be strongly inhomogeneous. For thick films the relaxation times were rather low in the middle of the film, whereas higher values were observed when approaching the substrate-polymer interface. In the latter case, the dynamics monotonically slowed down with increasing strength of adsorption $\epsilon_{s p}$. In the middle layers, which constitute the larger fraction of the film, the characteristic relaxation times $\tau_{\alpha}$, as presented in Figure 3, were fairly constant for $\epsilon_{s p} \leq 2$ but a shift to lower values was found for $\epsilon_{s p} \geq 3$, thus explaining the overall acceleration observed in Figure 2.

Increasing strength of adsorption resulted in a small decrease of density in the middle of the films, due to the fact that the films were simulated under NVT conditions. In order to ensure that this density difference is not mainly responsible for the faster dynamics observed at high values of $\epsilon_{s p}$ in the middle film-layers (Figure 4(a)), we repeated the layer-resolved analysis of the segmental orientational mobility for films created under NPT conditions. The external pressure was adjusted in order to have film thicknesses $D \approx 30$ for temperatures $T \approx 1$. The layer-resolved relaxation times (Figure 5(b)) showed similar non-monotonic behavior as found in NVT, which therefore could not be attributed only to density effects.

The effect of confinement was studied for different film thicknesses and strengths of adsorption to the substrates. Analysis of the layer-resolved segmental dynamics for the case of energetically neutral $\left(\epsilon_{s p}=1\right)$ substrates showed a strong shift to higher overall mobility with decreasing thickness (due to finite-size scaling effects ${ }^{14,18,20}$ ), but the same behavior was not found for stronger adsorption strengths. This was due to the thickness-dependent behavior of the middlelayer dynamics upon increasing $\epsilon_{s p}$, where for intermediate and high thicknesses the middle-layer mobility accelerates, but for low thicknesses it was hardly affected. The acceleration of the mobility could be attributed to the creation of glassy boundary layers with increasing $\epsilon_{s p}$, which resulted in an effective decrease of the thickness of the mobile middle layer and therefore, in an additional finite-size effect.

Film-averaged $\alpha$-relaxation times were extracted for all film thicknesses and for substrate attraction strengths $\epsilon_{s p}=1$ and $\epsilon_{s p}=10$, and compared with bulk values (Figure 7). It was shown that such average relaxation times are strongly influenced by layer-to-layer inhomogeneity in the mobility, in particular for strongly adsorbing substrates. As a result such relaxation times will depend on the chosen time interval.

A main conclusion of this work is that film-averaged segmental relaxation in capped films cannot be understood without resolving the inhomogeneous influence of substrate attraction and film thickness in space and time.

\section{ACKNOWLEDGMENTS}

This work is part of the research program of the Dutch Polymer Institute, Project No. 701. It was also sponsored by the Stichting Nationale Computerfaciliteiten (National Computer Facilities Foundation, NCF) for the use of supercomputer facilities, with financial support from the Nederlandse Organisatie voor Wetenschappelijk Onderzoek (Netherlands Organization from Scientific Research, NWO). We thank Daniel Bonn (University of Amsterdam, experimental part of the project) as well as the industrial partners Babak Hosseinkhani of SKF and Marc Couty of Michelin for helpful discussions.

${ }^{1}$ M. Park, C. Harrison, P. M. Chaikin, R. A. Register, and D. H. Adamson, Science 276, 1401-1404 (1997).

${ }^{2}$ D. C. Olson, J. Piris, R. T. Collins, S. E. Shaheen, and D. S. Ginley, Thin Solid Films 496, 26-29 (2006).

${ }^{3}$ L. M. Feller, S. Cerritelli, M. Textor, J. A. Hubbell, and S. G. P. Tosatti, Macromolecules 38, 10503-10510 (2005).

${ }^{4}$ P. Karagiannidis, D. Georgiou, C. Pitsalidis, A. Laskarakis, and S. Logothetidis, Mater. Chem. Phys. 129, 1207-1213 (2011).

${ }^{5}$ G. Heinrich and M. Klúppel, Filled Elastomers Drug Delivery Systems, Advances in Polymer Science, Vol. 160 (Springer, Berlin, Heidelberg, 2002), pp. $1-44$.

${ }^{6}$ G. Heinrich, M. Klúppel, and T. A. Vilgis, Curr. Opin. Solid State Mater. Sci. 6, 195-203 (2002).

${ }^{7}$ F. Saeed, A. Ansarifar, R. J. Ellis, Y. Haile-Meskel, and M. S. Irfan, J. Appl. Polym. Sci. 123, 1518-1529 (2012).

${ }^{8}$ K. W. Stôckelhuber, A. S. Svistkov, A. G. Pelevin, and G. Heinrich, Macromolecules 44, 4366-4381 (2011).

${ }^{9}$ J. L. Keddie and R. A. L. Jones, Europhys. Lett. 27, 59-64 (1994).

${ }^{10}$ J. L. Keddie, R. A. L. Jones, and R. A. Cory, Faraday Discuss. 98, 219-230 (1994).

${ }^{11}$ J. Forrest, K. Dalnoki-Veress, and J. Dutcher, Phys. Rev. E 56, 5705 (1997).

${ }^{12}$ C. L. Soles, J. F. Douglas, W.-L. Wu, H. Peng, and D. W. Gidley, Macromolecules 37, 2890-2900 (2004).

${ }^{13}$ R. Inoue, T. Kanaya, K. Nishida, I. Tsukushi, M. T. F. Telling, B. J. Gabrys, M. Tyagi, C. Soles, and W.-L. Wu, Phys. Rev. E 80, 031802 (2009).

${ }^{14}$ F. Varnik, J. Baschnagel, and K. Binder, Phys. Rev. E 65, 021507 (2002).

${ }^{15}$ J. A. Torres, P. F. Nealey, and J. J. de Pablo, Phys. Rev. Lett. 85, 3221-3224 (2000).

${ }^{16}$ L. Yelash, P. Virnau, K. Binder, and W. Paul, Phys. Rev. E 82, 050801 (2010).

${ }^{17}$ D. Hudzinskyy, A. V. Lyulin, A. R. C. Baljon, N. K. Balabaev, and M. A. J. Michels, Macromolecules 44, 2299-2310 (2011).

${ }^{18}$ C. Batistakis, A. V. Lyulin, and M. A. J. Michels, Macromolecules 45, 7282-7292 (2012).

${ }^{19}$ K. Johnston and V. Harmandaris, Soft Matter 8, 6320-6332 (2012).

${ }^{20}$ G. Adam and J. H. Gibbs, J. Chem. Phys. 43, 139-146 (1965).

${ }^{21}$ D. van der Spoel, E. Lindahl, B. Hess, A. R. van Buuren, E. Apol, P. J. Meulenhoff, D. P. Tieleman, A. L. T. M. Sijbers, K. A. Feenstra, R. van Drunen, and H. J. C. Berendsen, Gromacs user manual version 4.5, see www.gromacs.org (2010).

${ }^{22}$ B. Hess, H. Bekker, H. J. C. Berendsen, and J. G. E. M. Fraaije, J. Comput. Chem. 18, 1463-1472 (1997).

${ }^{23}$ B. Hess, J. Chem. Theory Comput. 4, 116-122 (2008).

${ }^{24}$ W. Humphrey, A. Dalke, and K. Schulten, "VMD: visual molecular dynamics," J. Mol. Graphics 14, 33-38 (1996).

${ }^{25}$ E. Leutheusser, Phys. Rev. A 29, 2765-2773 (1984)

${ }^{26}$ J. P. Hansen, D. Levesque, and J. Zinn-Justin, Liquids, Freezing and the Glass Transition (North-Holland, 1991).

${ }^{27}$ F. Varnik, J. Baschnagel, and K. Binder, Eur. Phys. J. E 8, 175-192 (2002).

${ }^{28}$ G. Williams and D. C. Watts, Trans. Faraday Soc. 66, 80-85 (1970).

${ }^{29}$ A. V. Lyulin, N. K. Balabaev, and M. A. J. Michels, Macromolecules 35, 9595-9604 (2002).

${ }^{30}$ A. van Zon and S. W. de Leeuw, Phys. Rev. E 60, 6942-6950 (1999).

${ }^{31}$ B. Vorselaars, A. V. Lyulin, and M. A. J. Michels, Macromolecules 40, 6001-6011 (2007) 